\title{
Evidence of Platelet Activation Associated with Establishment of Pregnancy in Cows with Transferred Embryos
}

\author{
Toshiyuki KOJIMA\#, Satoshi AKAGI\#\#, Yumie ZENIYA, \\ Manabu SHIMIZU and Tsuneo TOMIZUKA
}

Department of Animal Reproduction, National Institute of Animal Industry, Tsukuba-Norindanchi, P.O.Box 5, Ibaraki 305, Japan

\begin{abstract}
The aims of the present study were twofold. The first aim was to examine whether or not early pregnancy-associated thrombocytopenia (EPAT) occurred in cows following nonsurgical embryo transfer by observing the daily patterns of the concentration of peripheral platelets (PLT) during the preimplantation period of pregnancy. The other goal was to assess the use of PLT count as a diagnosis of early pregnancy in cows. In Experiment 1, out of 50 Holstein parous cows, 40 had one embryo nonsurgically transferred to each uterine horn on Day 6 or 7 (Day $0=$ onset of standing estrus). The remaining 10 cows served as controls having no embryos transferred. Blood was taken daily at the same time, from Day 6 or 7 following estrus (the day when the embryos were transferred in the non-controls) to the day of return to estrus for control cows and non-pregnant cows, or to Day 36 for pregnant cows. Bilateral embryo transfers resulted in 9 twin, 14 single, and 17 nonpregnancies. Some recipient cows showed an increase in PLT following embryo transfer, while others displayed a decrease in PLT. If significant PLT change was characterized either by a decrease or an increase of $>20 \%$ compared to the value of pre-transfer, the proportions of cows indicating significant change were $78 \%(7 / 9)$ in twin, $64 \%(9 / 14)$ in single, $76 \%(13 / 17)$ in nonpregnancies and $30 \%(3 / 10)$ in controls. The proportions in twin $(\mathrm{P}<0.05)$, single $(\mathrm{P}<0.05)$ and nonpregnancies $(\mathrm{P}<0.01)$ were higher than that in controls. The effect of the particular group on PLT was significant $(\mathrm{P}<0.0025)$. In Experiment 2, each of several in vitro-produced blastocysts were nosurgically transferred to 9 Japanese Black cows on Day 7 or 8 following onset of estrus. Seven days after embryo transfer, an attempt to recover the previously transferred embryos by nonsurgical flushing was performed. Embryos could be recovered on Day 14 or 15 from 5 animals, whose blood was taken from the day of embryo transfer to the next day of embryo recovery and during the corresponding days of the following estrous cycle. For PLT, differences were detected between the both phases of 3 out of the 5 cows. The individual PLT variation in response means that EPAT does not necessarily occur in cows following embryo transfer, and that the use of PLT counts is unlikely to be clinically useful in assessing early pregnancy in cows. These results may suggest, however, that maternal platelet activation would occur during early pregnancy in cattle.

Key words: Platelet concentration, Pregnancy establishment, Cow, Platelet activation, Embryo transfer.
\end{abstract}

(J. Reprod. Dev. 42: 225-235, 1996)

Accepted for publication: July 12, 1996

Correspondence: T. Kojima

\#Present address: National Livestock Breeding Center, Ministry of Agriculture, Forestry and Fisheries, Odakurahara-1, Nishigoh, Nishishirakawa, Fukushima 961, Japan

\#\#Present address: Kyushu National Agricultural Experiment Station Nishigoshi-machi, Kumamoto 861-11, Japan
E arly pregnancy-associated thrombocytopenia E (EPAT), which is characterized by a sustained decline in peripheral platelet counts (PLT), related to the presence of fertilized eggs has been demonstrated in mice [1-3]. EPAT may be the earliest 
physiological change associated with pregnancy in the maternal body, and may reflect maternal platelet activation. This maternal physiological alteration appears to be universal. Preliminary results have suggested that EPAT caused by embryonic product also occurs in humans $[4,5]$ and marmoset monkeys [6]. In a study of in vitro fertilization and embryo transfer, $\mathrm{O}^{\prime}$ Neill et al. [4] demonstrated that only the embryos producing platelet-activating factor (PAF) in vitro caused EPAT after embryo transfer and only patients displaying EPAT had a successful pregnancy established. Furthermore, EPAT was not a consequence of the activation of the maternal blood coagulation cascade or of maternal immunologic recognition of the embryo [2]. Therefore, while detection of EPAT may be beneficial to monitor the embryonic survival in the reproductive tract and to assess pregnancy potential, the physiological significance of EPAT is not clear.

Although several experiments have been conducted to determine EPAT in cattle [7-9], there has been conflicting evidence for its occurrence in inseminated cows. This inconsistency may be due to the facts that individual PLT concentrations in cows are highly variable and inseminated cows in the previous studies suffered losses associated with fertilization failure and aberrant embryonic development prior to Day 7. In humans, not all pregnancies resulting from gamete intrafallopian transfer has been associated with a fall in PLT [10]. As embryo transfer itself can markedly reduce the losses associated with fertilization and aberrant embryonic development prior to Day 7, it should be recognized that factors associated with these losses are originally excluded from an experiment based on embryo transfer technique.

The present study was designed to examine whether or not EPAT occurs in cows following nonsurgical embryo transfer by observing the daily concentration patterns of PLT during the preimplantation period of pregnancy and to assess the use of PLT count as a diagnosis of early pregnancy in cows.

\section{Materials and Methods}

\section{Experiment 1}

The aim of Experiment 1 was to examine the daily patterns of concentrations of both PLT and peripheral red blood cell (RBC) during the preimplantation period of pregnancy in cows following bilateral embryo transfer. Fifty regularly cycling Holstein cows of varying age and parity, which were housed, fed and managed in National Institute of Animal Industry were used as recipient animals for the present study. Estrus was checked twice a day using a heat mount detector (Kamar Inc., CO, USA). Out of 50 cows, 40 had one embryo nonsurgically transferred to each uterine horn on Day 6 or 7 (Day $0=$ onset of standing estrus). The remaining 10 cows, which were checked for estrus but did not receive any embryos, served as controls.

Either expanded blastocysts, which were derived from in vitro maturation and in vitro fertilization (fresh-vitro embryos); late morulae to expanded blastocysts, which were recovered from superovulated Japanese Black heifers (fresh-vivo embryos) on Day 7 or 8; or frozen-thawed fresh-vivo embryos (frozen-vivo embryos) were used for bilateral transfer.

Fresh-vitro embryos used in the present study were obtained from a commercial supplier and their production details are described elsewhere [11]. Ovaries were obtained from a local abattoir and transported to the laboratory. All visible follicles (2 to $5 \mathrm{~mm}$ in diameter) were aspirated. Cumulus-oocyte complexes were cultured for $21 \mathrm{~h}$ in a maturation medium. After maturation culture, the cumulus-oocyte complexes were introduced into the drops of sperm suspension. They were cultured together for $5 \mathrm{~h}$. Forty-eight hours after insemination, the oocytes were removed from the cumulus cell masses and then examined for their cleavage stages; 4 - to 8-cell embryos were further cultured with a cumulus cell monolayer. Embryos that developed to the blastocyst and expanded blastocyst stages after 7 days were used for the present experiment.

The superovulatory regimen to obtain fresh-vivo embryos is given elsewhere [12]. Briefly, donors received FSH (Antrin, Denka Pharmaceutical Ltd., Kawasaki, Japan) twice daily in decreasing doses of $5,5,4,4,3,3 \mathrm{mg}$ for three consecutive days, beginning on Days 9 to 12 of the same estrous cycle. All of the donors were given $15 \mathrm{mg}$ of $\mathrm{PGF}_{2} \alpha$ (Panaceran-Hi, Daiichi Pharmaceutical Ltd., Tokyo, Japan) at 48 and $60 \mathrm{~h}$ after the initiation of the superovulatory treatment. The superovulated-do- 
nors were artificially inseminated with one unit of frozen semen $24 \mathrm{~h}$ after the onset of standing estrus. Embryos were recovered nonsurgically on Day 7 or 8 and morphologically evaluated.

A proportion of the nonsurgically recovered embryos were frozen using the following procedure (frozen-vivo embryos): Within a few hours after embryo collection, embryos assessed as having excellent or good morphology were equilibrated with $1.36 \mathrm{M}$ glycerol in PBS solution supplemented with $10 \%$ fetal calf serum (FCS). After $10 \mathrm{~min}$, the embryos were moved to a freezing solution (1.36 M glycerol containing $0.2 \mathrm{M}$ trehalose) and loaded into a $0.25 \mathrm{ml}$ plastic straw (A201, I.M.V., L'Aigle, France) within $5 \mathrm{~min}$. The straws containing embryos were immersed directly into methanol and cooled at $-7 \mathrm{C}$ in the bath of a programmable freezer (EFP-40, EYELA, Tokyo Rikakikai, Tokyo, Japan). Seeding was performed by the silver iodide droplet method [13]. After $10 \mathrm{~min}$ at $-7 \mathrm{C}$, the temperature of the bath was decreased at the rate of $-0.33 \mathrm{C} /$ min to $-25 \mathrm{C}$, followed by plunging into liquid nitrogen. The frozen embryos were prepared for transfer by air thawing and then rehydrating in a two-step process of bathing in $0.5 \mathrm{M}$ trehalose and washing in Celgrosser- $\mathrm{H}^{\circledR}$ (Sumitomo Pharmaceutical Co., Ltd., Osaka, Japan) at room temperature.

The embryos, each of which was loaded into $0.25 \mathrm{ml}$ plastic straw using Celgrosser- $\mathrm{H}^{\circledR}$ containing $0.1 \% \mathrm{BSA}$, were bilaterally transferred to the uterine horns of recipient cows on Days 5 to 8 after estrus using the nonsurgical transcervical method. Each cow was palpated to determine the presence and location of corpus luteum prior to transfer. Following embryo transfer, all recipient cows were checked for return of estrus. Pregnancy was diagnosed using a real-time ultrasonographer (Aloca-650, Aloca, Tokyo, Japan) with a $5 \mathrm{MHz}$ rectal transducer on Day 30. Twin pregnancies were diagnosed between Days 30 and 60.

Daily blood collection ( $2 \mathrm{ml}$ ) using jugular venipuncture into a siliconized plastic bottle containing EDTA-2K (Erma, Tokyo, Japan) was commenced on the morning of embryo transfer and continued until either the day of return to estrus for both control cows (non-bred and non-transferred) and non-pregnant cows or to Day 36 for pregnant cows. Blood was taken and kept at room temperature, and the enumeration of PLT, RBC and measurement of hematocrit (HCT) were performed with duplication within an hour after collection using a semiautomatic blood cell counter (PC-608, Erma, Tokyo, Japan). PLT, RBC and HCT values on the day when the embryos were transferred were designated as 100 (pre-transfer). Therefore, PLT, RBC and HCT on subsequent days were relative values unless otherwise stated.

\section{Experiment 2}

The aim of Experiment 2 was to confirm evidence of platelet activation and hematopoietic system stimulation by the presence of viable embryos (Days 7 to 14 or Days 8 to 15), by comparing the phase in which embryos existed in uterus and the control phase of the same Japanese Black cows. In Experiment 2, each of several fresh-vitro embryos at the blastocyst stage were nonsurgically transferred into the uterine horn ipsilateral to the corpus luteum in: (a) three normal parous cows aging 3 to 5 years old; (b) three normal nulliparous heifers aging 10 to 15 months old; or (c) three repeat breeder-heifers aging 2 to 3 years old on Day 7 or 8 after estrus. Seven days after embryo transfer, an attempt to recover the embryos previously transferred by nonsurgical uterine flushing with $1,000 \mathrm{ml}$ PBS containing $0.1 \%$ BSA was undertaken. Cows from which at least one elongated blastocysts were obtained were designated pregnant. Cows from which no embryo was recovered were treated as non-pregnant, and data from the cows were not used for analysis. Daily blood sampling (pregnant phase) was performed from these day of embryo transfer to the next day of embryo recovery as in Experiment 1 . Daily blood sampling (control phase) was also carried out for the cows from which elongated blastocysts were recovered during the corresponding days of the following estrous cycle. The values of each cow during the corresponding days of the following estrous cycle acted as controls. No replication of the two phases (pregnant phase and control phase) was performed for each cow.

\section{Statistical analysis}

The general linear models procedure of the Statistical Analysis System (SAS ${ }^{\circledR}$ ) [14] with least square means was used to analyze the effect of pregnancy on PLT, RBC and HCT during the preimplantation period. The model was as follows: 

where

$\mathrm{Y}_{\mathrm{ijk}}=\mu+\mathrm{G}_{\mathrm{i}}+\mathrm{D}_{\mathrm{j}}+\mathrm{C}_{(\mathrm{i}) \mathrm{k}}+\mathrm{GD}_{\mathrm{ij}}+\mathrm{e}_{\mathrm{ijk}}$

$\mu=$ overall means,

$\mathrm{G}_{\mathrm{i}}=$ effect of group (pregnant states),

$D_{j}=$ effect of sampling day after embryo transfer,

$\mathrm{C}_{(\mathrm{i}) \mathrm{k}}=$ random variable cow, nested in group,

$\mathrm{GD}_{\mathrm{ij}}=$ interactions, and

$\mathrm{e}_{\mathrm{ijk}}=$ residuals.

Other data were analyzed by the Chi Square test. Significant difference was declared at $\mathrm{P}<0.05$.

\section{Results}

\section{Experiment 1}

Bilateral embryo transfers to 40 recipient cows resulted in 9 twin pregnancies, 14 single pregnancies, and 17 non-pregnancies, judging from the diagnosis performed during Days 30 to 60 after estrus. The detailed results about embryo survival following the bilateral embryo transfers were reported elsewhere [15]. Briefly, no significant difference in embryonic survival rates was observed between the origin of embryos transferred. Therefore the origin of embryos did not greatly affect results in the present study.

Figures 1-3 show daily patterns of the mean relative concentrations of PLT, daily patterns of the mean relative concentrations of $\mathrm{RBC}$, and daily patterns of the mean relative values of HCT in four groups consisting of twin, single pregnant, nonpregnant or control cows respectively. The significances were not shown in these figures to avoid over-complicated indications. Significant group effect of pregnancy status on relative PLT was detected during the preimplantation period $(\mathrm{P}<0.0025)$. There were significant differences between twin pregnant cows and control cows $(\mathrm{P}<0.0001)$, between single pregnant cows and controls $(\mathrm{P}<0.0001)$ and between non-pregnant cows and controls $(\mathrm{P}<0.0001)$. Significant differences were observed between twin pregnant cows and single pregnant cows on Day 8 after embryo transfer $(\mathrm{P}<0.025)$, on Day $9(\mathrm{P}<0.05)$ and on Day 23 $(\mathrm{P}<0.025)$. Also, there were significant differences between single pregnant and controls on Day 6 after embryo transfer $(\mathrm{P}<0.01)$ and on Day 13 $(\mathrm{P}<0.05)$. Similarly, between twin pregnancies and controls there were differences on Day $7(\mathrm{P}<0.05)$, Day $8(\mathrm{P}<0.0025)$, Day $10(\mathrm{P}<0.025)$ and Day 15 $(\mathrm{P}<0.05)$. Between twin pregnancies and non-preg-

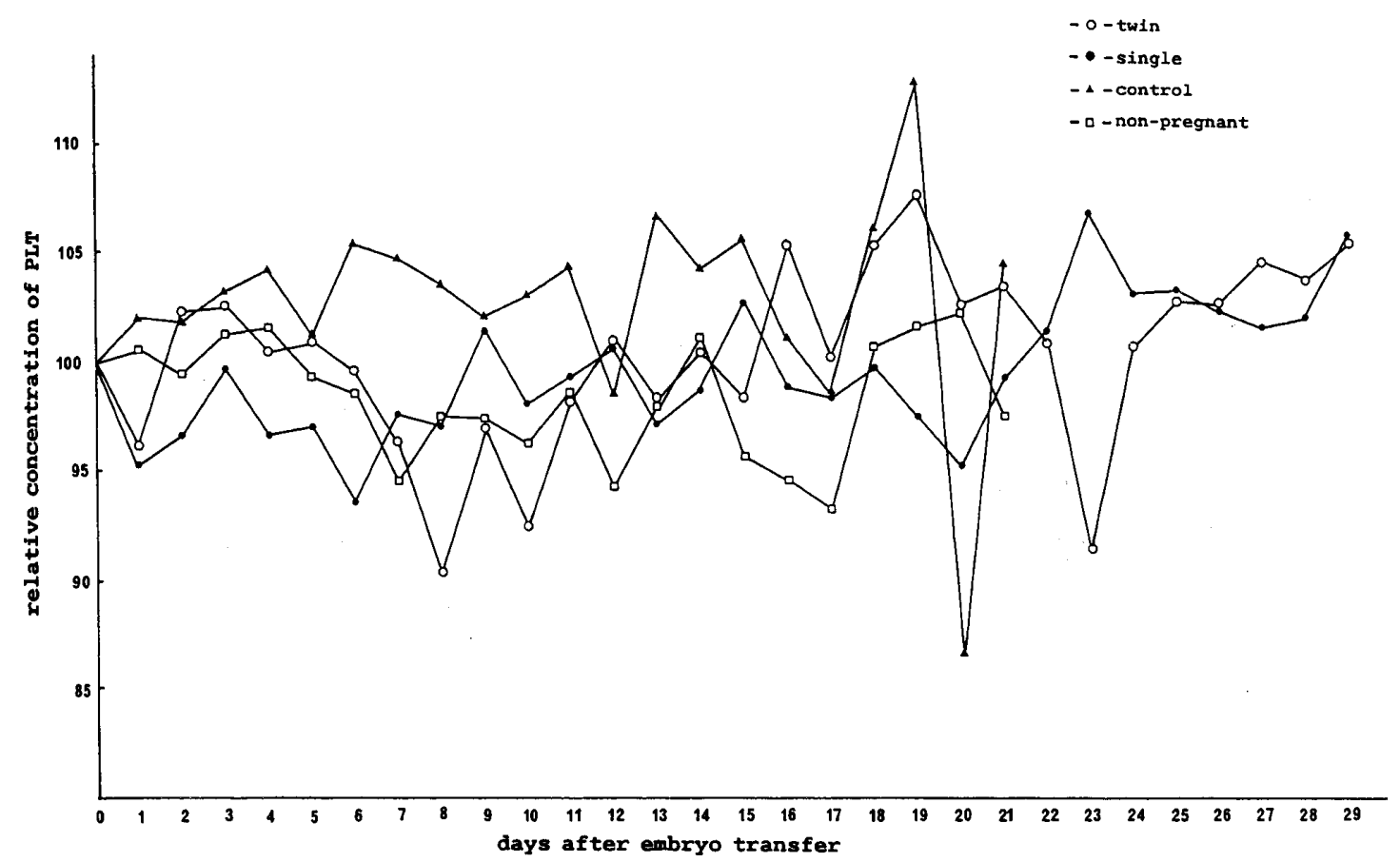

Fig. 1. Daily changes in the mean relative concentrations of peripheral platelets in twin pregnant (- $\bigcirc-)$, single pregnant (- -$)$, control $(-\mathbf{\Delta}-)$ ) and non-pregnant $(-\square-)$ recipient cows. 


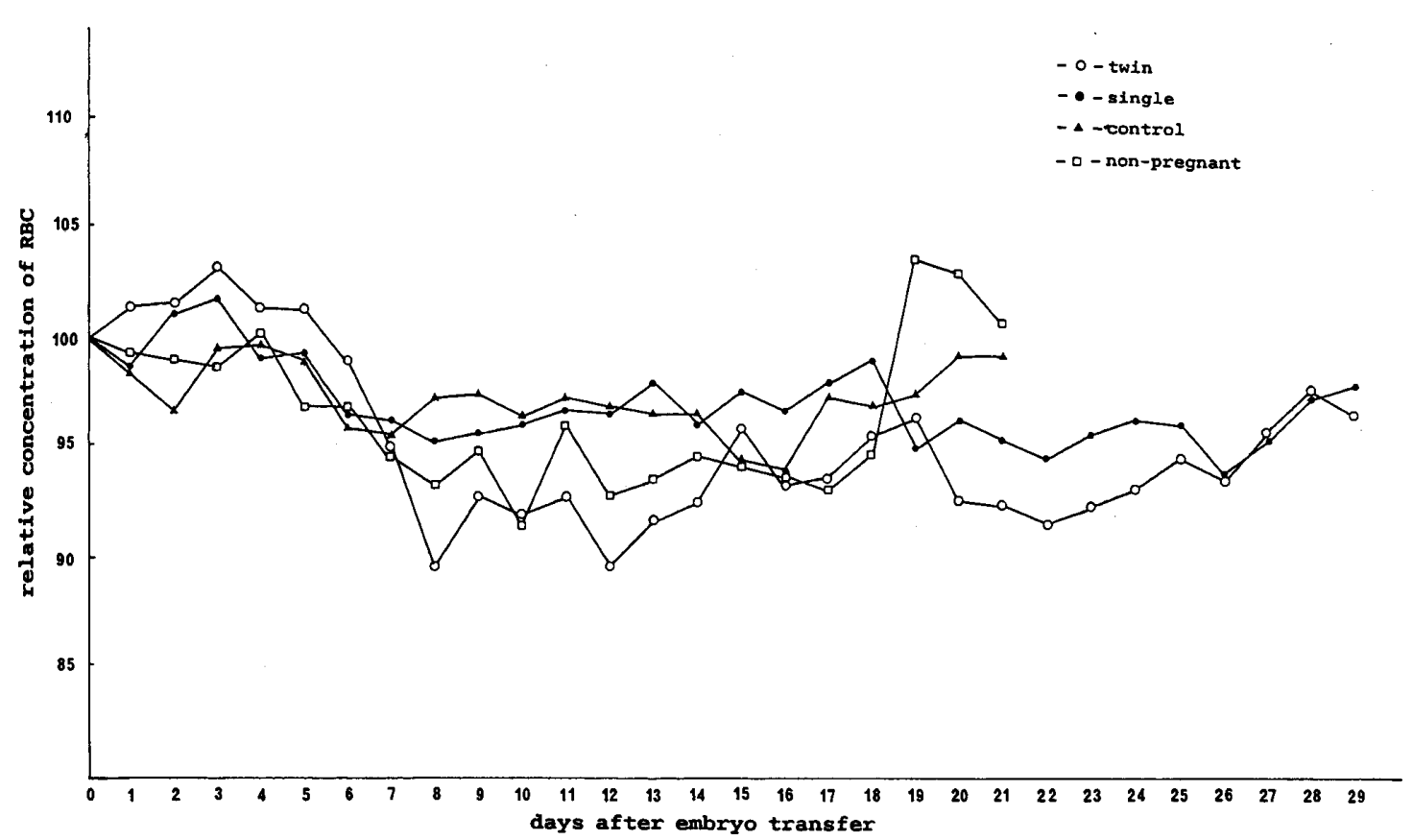

Fig. 2. Daily changes in the mean relative concentrations of peripheral red blood cell in twin pregnant

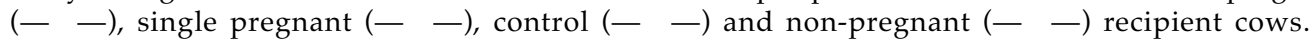

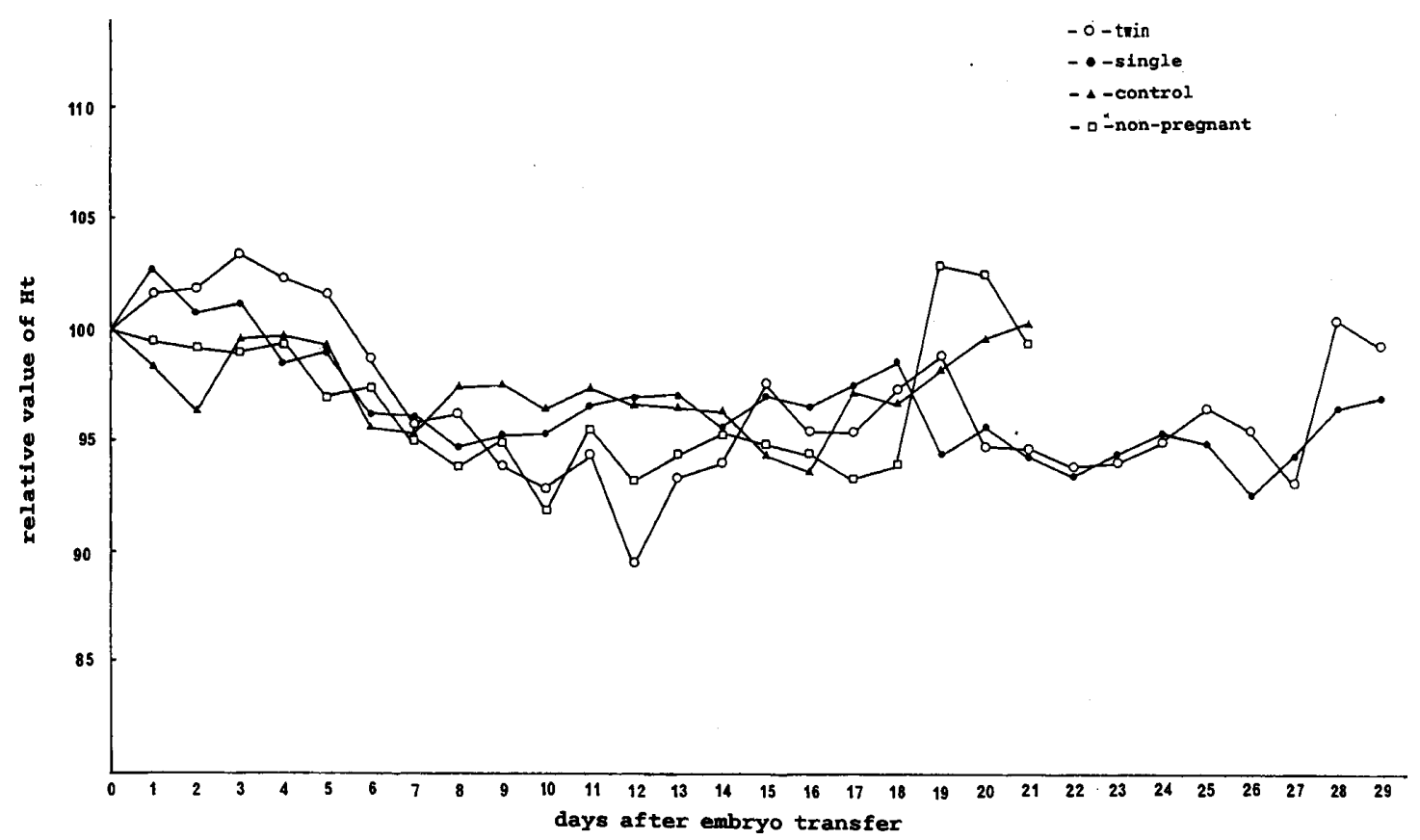

Fig. 3. Daily changes in the mean relative values of hematocrit in twin pregnant (- $\bigcirc-)$, single pregnant (一-), control (- $-\mathbf{\Delta})$ and non-pregnant $(-\square-)$ recipient cows. 
nancies, significant differences were observed on Day $8(\mathrm{P}<0.05)$ and Day $16(\mathrm{P}<0.05)$. Between single pregnancies and non-pregnancies, a difference was seen on Day $15(\mathrm{P}<0.05)$. Between controls and non-pregnancies, differences were detected on Day $7(\mathrm{P}<0.01)$, on Day $13(\mathrm{P}<0.05)$ and on Day 15 $(\mathrm{P}<0.01)$.

For $\mathrm{RBC}$, the effects of group and day were respectively significant $(\mathrm{P}<0.0001)$. Significant differences were observed between twin and single pregnancies $(\mathrm{P}<0.0001)$, between twin pregnancies and controls $(\mathrm{P}<0.0005)$, between single pregnancies and controls $(\mathrm{P}<0.005)$, between single pregnancies and non-pregnancies $(\mathrm{P}<0.0001)$ and between controls and non-pregnancies $(\mathrm{P}<0.05)$. In detail, between twin and single pregnancies there were differences on Days $8(\mathrm{P}<0.001)$, $9(\mathrm{P}<0.025), 11(\mathrm{P}<0.025), 12(\mathrm{P}<0.025), 13(\mathrm{P}<0.005)$, $14(\mathrm{P}<0.025), 15(\mathrm{P}<0.05), 16(\mathrm{P}<0.05), 20(\mathrm{P}<0.05)$, $21(\mathrm{P}<0.025), 22(\mathrm{P}<0.05), 23(\mathrm{P}<0.05), 24(\mathrm{P}<0.025)$ and $29(\mathrm{P}<0.05)$. Between twin pregnancies and controls differences were detected on Days 8 $(\mathrm{P}<0.001)$ and $9(\mathrm{P}<0.05)$. Between twin pregnancies and non-pregnancies differences were seen on Days $20(\mathrm{P}<0.025), 22(\mathrm{P}<0.05)$ and $25(\mathrm{P}<0.05)$. A difference was observed on Day $2(\mathrm{P}<0.025)$ between single pregnancies and controls, and on Days $3(\mathrm{P}<0.05), 10(\mathrm{P}<0.025), 12(\mathrm{P}<0.05), 13(\mathrm{P}<0.01)$, $15(\mathrm{P}<0.025), 16(\mathrm{P}<0.05)$ and $17(\mathrm{P}<0.05)$ between single pregnancies and non-pregnancies. A differ- ence was seen on Day $10(\mathrm{P}<0.05)$ between controls and non-pregnancies.

For HCT, like RBC, the effects of group $(\mathrm{P}<0.025)$ and day $(P<0.0001)$ were significant. Significant differences were detected between twin pregnancies and single pregnancies $(\mathrm{P}<0.01)$, between single pregnancies and controls $(\mathrm{P}<0.05)$, and between single pregnancies and non-pregnancies $(\mathrm{P}<0.0001)$. There were significant differences on Days 1 $(\mathrm{P}<0.05)$ and $2(\mathrm{P}<0.025)$ after embryo transfer in HCT between single pregnancies and controls, and Days $12(\mathrm{P}<0.05)$ and $13(\mathrm{P}<0.05)$ between single pregnancies and non-pregnancies. A significant difference was seen on Day $10(\mathrm{P}<0.05)$ between controls and non-pregnancies.

Figure 4 demonstrates distribution maps indicating the correlations between RBC and PLT in twin pregnant, single pregnant, control and nonpregnant recipient cows. There were the significant positive correlations in twin pregnancies $(\mathrm{r}=0.48$, $\mathrm{P}<0.0001)$ and in single pregnancies $(r=0.43$, $\mathrm{P}<0.0001$ ), while no significances were detected in controls ( $\mathrm{r}=0.0006, \mathrm{P}=0.297)$ and in non-pregnancies $(\mathrm{r}=0.09, \mathrm{P}=0.247)$. When the correlations during two separate stages (Days 0 to 14 and Days 15 to 29) of early pregnancy were calculated, the respective correlations in both twin $(\mathrm{r}=0.32, \mathrm{P}<0.0005$; $\mathrm{r}=0.23, \mathrm{P}<0.025)$ and single pregnant $(\mathrm{r}=0.30$, $\mathrm{P}<0.0001 ; \mathrm{r}=0.62, \mathrm{P}<0.0001)$ cows were significant. The proportion of cows indicating significant re-
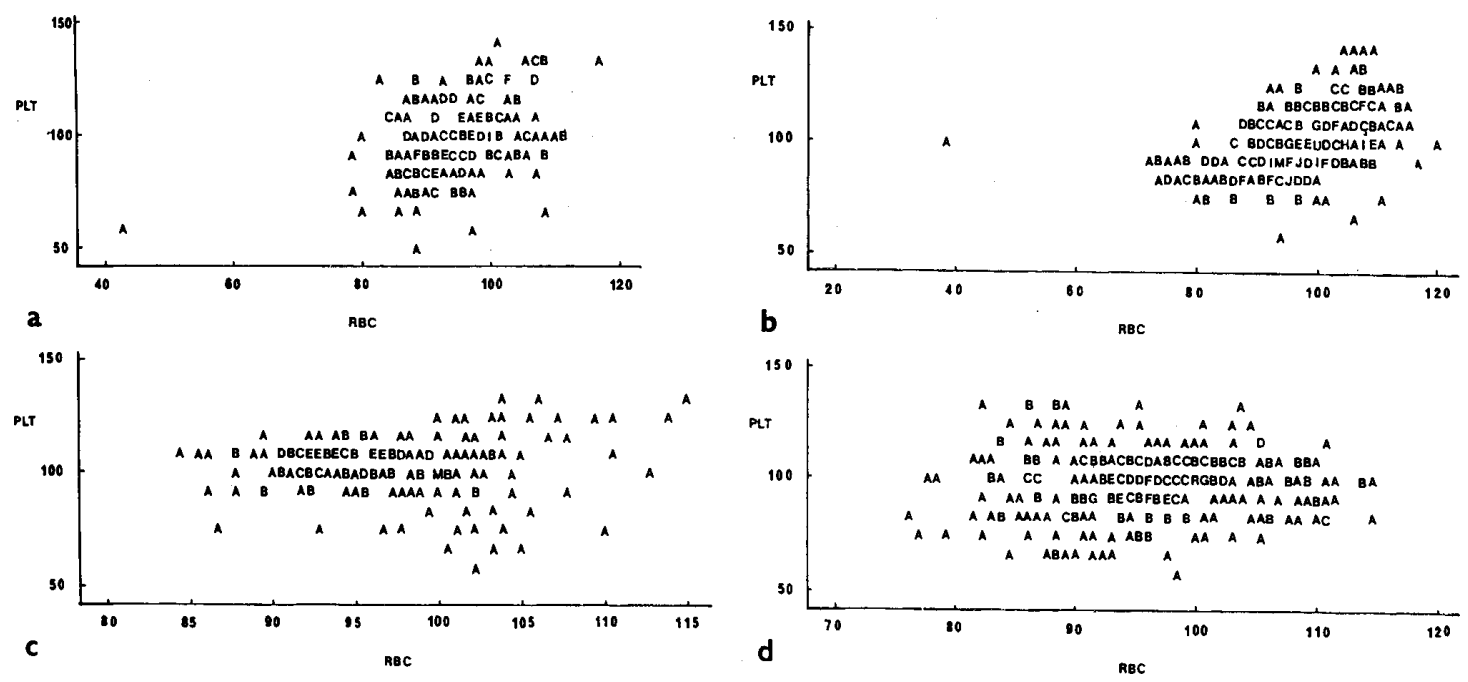

Fig. 4. Distribution maps indicating the correlation between relative red blood cell concentrations and relative platelet concentrations in twin pregnant (a), single pregnant (b), control (c) and non-pregnant (d) recipient cows. A indicates one observation, $B$ two observations and so forth. a: $r=0.48, P<0.0001$; b: $\mathrm{r}=0.43, \mathrm{P}<0.0001 ; \mathrm{c}: \mathrm{r}=0.0006, \mathrm{P}=0.297 ; \mathrm{d}: \mathrm{r}=0.09, \mathrm{P}=0.247$. Day 0 indicates the day of embryo transfer. 
gression between RBC and PLT in each pregnancy status was examined. The proportions in twin (89\%, 8/9; $\mathrm{P}<0.01)$, single $(71 \%, 10 / 14 ; \mathrm{P}<0.05)$ and non-pregnancies $(65 \%, 11 / 17 ; \mathrm{P}<0.05)$ were higher than that in controls $(20 \%, 2 / 10)$.

When regression analysis between the thrombocrit $\left(=\mathrm{PLT} \times\right.$ mean platelet volume; $\left.\mu \mathrm{m}^{3} \times 10^{6} / \mu \mathrm{l}\right)$ at pre-transfer and the relative PLT change following embryo transfer was performed (Fig. 5), the slopes of the regression lines obtained were positive in contrast with the results of $\mathrm{O}^{\prime} \mathrm{Neill}$ et al. [16].

\section{Experiment 2}

The embryo recovery rates from recipient cows

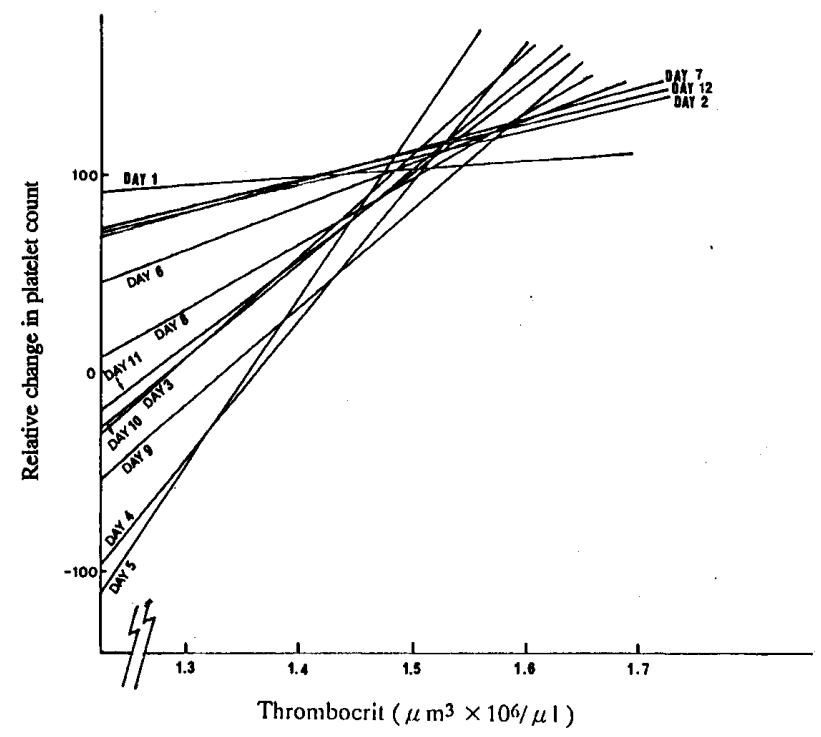

Fig. 5. Regression lines between thrombocrit on Day 0 and each platelet concentration after embryo transfer (from Day 1 to Day 12). Day 0 indicates the day of embryo transfer. Thrombocrit=platelet concentration $\left(10^{6} / \mu \mathrm{l}\right) \times$ mean volume of platelet $\left(\mu \mathrm{m}^{3}\right)$. which had transferred bovine embryos produced in vitro are shown in Table 1 and are compared in relation to the reproductive states. While normal elongated blastocysts were successfully recovered at the rate of $100 \%(4 / 4)$ from the normal parous cows and at the rate of $67 \%(2 / 3)$ from the normal nulliparous heifers, no embryos were recovered in four trials from three repeat breeder heifers. Efficiencies of embryo recovery were 19\% $(5 / 27)$ from normal parous cows and $17 \%(4 / 24)$ from normal nulliparous heifers. Normal elongated blastocysts were recovered from 5 cattle, and these 5 cattle were used to analyze daily patterns of PLT, RBC and HCT from the day of embryo transfer to the next day of embryo recovery as pregnant cattle. No embryos could be recovered from the remaining 4 cattle ( 1 normal nulliparous heifer and 3 repeat breeder-heifers). Data from these 4 heifers were not used for subsequent statistical analyses.

Figures 6 and 7 demonstrate the daily changes of PLT and RBC from the five individual cattle, respectively, both during the phase of pregnancy and the phase of control. The effect of the random variable cow nested in the group was significant $(\mathrm{P}<0.0001)$. In $\mathrm{PLT}$, significant differences were detected between both phases of cow B $(\mathrm{P}<0.0001)$, cow $\mathrm{C}(\mathrm{P}<0.0001)$ and cow $\mathrm{E}$ $(\mathrm{P}<0.0025)$. The effect of the random variable cow nested in the group was significant $(\mathrm{P}<0.0001)$. For RBC, differences were observed between the both phases of cow A $(\mathrm{P}<0.0001)$, cow $\mathrm{B}(\mathrm{P}<0.025)$, cow $\mathrm{C}(\mathrm{P}<0.0001)$, cow $\mathrm{D}(\mathrm{P}<0.005)$ and cow $\mathrm{E}$ $(\mathrm{P}<0.0001)$. Like RBC, there were differences in individual HCT between the both phases of the five cows, cow $\mathrm{A}(\mathrm{P}<0.0001)$, cow $\mathrm{B}(\mathrm{P}<0.001)$, cow $\mathrm{C}(\mathrm{P}<0.025)$, cow $\mathrm{D}(\mathrm{P}<0.001)$ and cow $\mathrm{E}$ $(\mathrm{P}<0.0001)$.

Table 1. Comparison of embryo recovery rates from recipient cows having transferred bovine embryos produced in vitro, in relation to the reproductive states

\begin{tabular}{lcccc}
\hline & N & $\begin{array}{c}\text { No. of } \\
\text { recovery } \\
\text { trials }\end{array}$ & $\begin{array}{c}\text { No. of } \\
\text { successful } \\
\text { recoveries }\end{array}$ & $\begin{array}{c}\text { Efficiency of } \\
\text { embryo } \\
\text { recovery, \% }\end{array}$ \\
\hline Normal parous cows & 3 & 4 & 4 & $19(5 / 27)$ \\
Normal nulliparous heifers & 3 & 3 & 2 & $17(4 / 24)$ \\
Repeat breeder heifers & 3 & 4 & 0 & $0(0 / 23)$ \\
\hline
\end{tabular}



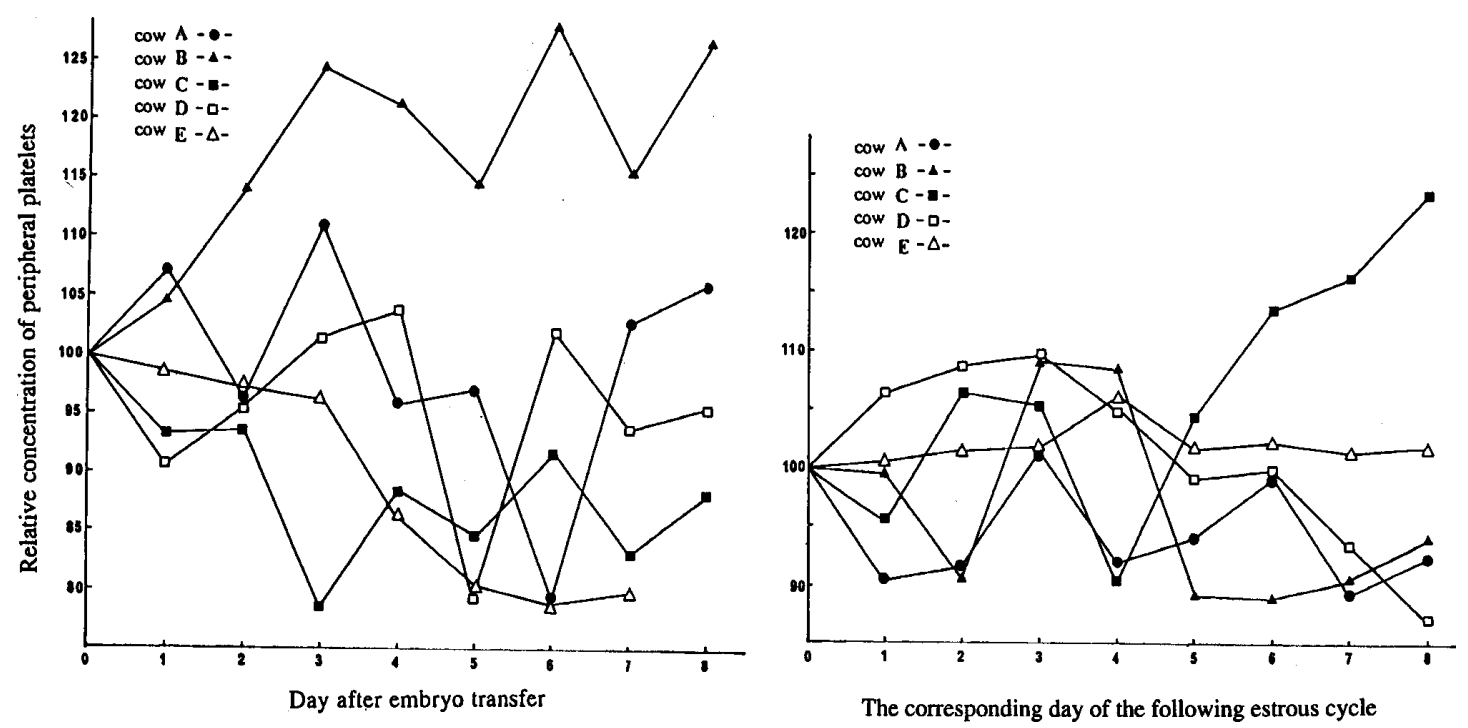

Fig. 6. Individual daily changes of peripheral platelet concentrations in cows (a) with transferred embryos, some of which were recovered after one week of embryo transfer, and in the same cows (b) during the corresponding days of the following estrous cycle after embryo collection.
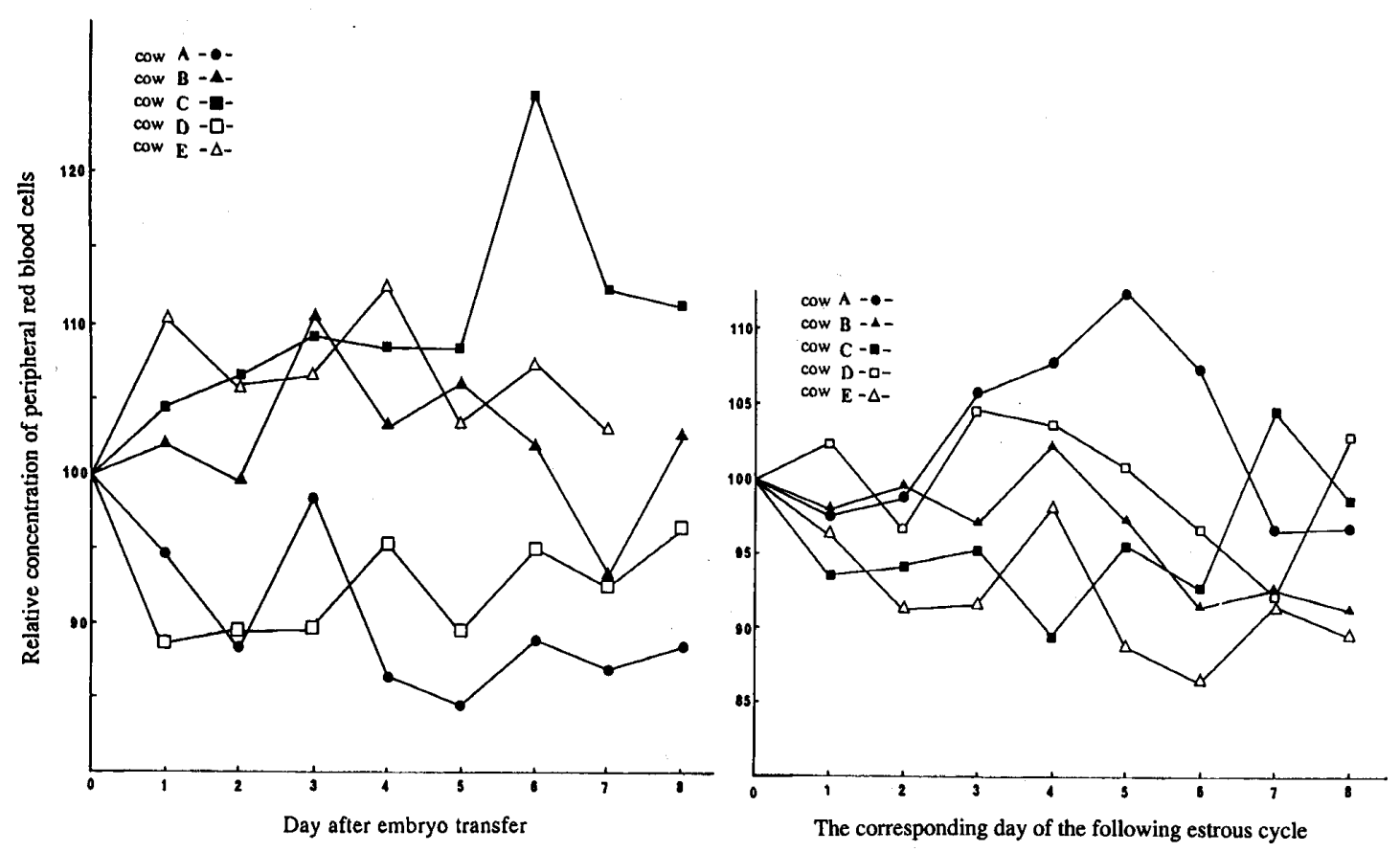

Fig. 7. Individual daily changes of peripheral red blood cell concentrations in cows (a) with transferred embryos, some of which were recovered after one week of embryo transfer, and in the same cows (b) during the corresponding days of the following estrous cycle after embryo collection. 


\section{Discussion}

The results obtained from the present study in cows having bilaterally transferred embryos demonstrated that not all pregnancies were associated with a sustained fall in PLT. However, some pregnant cows $(43 \%, 10 / 23)$ showed a transient decrease in PLT, others $(26 \%, 6 / 23)$ exhibited a transient increase in PLT. The remaining seven pregnant cows $(30 \%)$ showed a relatively stable pattern. Similarly, in the non-pregnant group of 17, 5 cows $(29 \%)$ showed a transient rise, 8 cows $(47 \%)$ a temporary fall and 4 cows $(24 \%)$ a stable pattern in PLT. On the other hand, in the controls 7 out of 10 cows $(70 \%)$ exhibited a stable pattern in PLT. However, most PLT patterns became stable by the following day. RBC and HCT showed a more stable pattern than PLT in 4 groups. If significant PLT change was characterized either by a decrease or by an increase of $>20 \%$ compared to the value of pre-transfer, the proportions of cows indicating significant change in PLT were 78\% (7/9) in twin pregnancies, $64 \%(9 / 14)$ in single pregnancies, $76 \%$ $(13 / 17)$ in non-pregnancies and $30 \%(3 / 10)$ in controls. The proportions in twin $(\mathrm{P}<0.05)$, single $(P<0.05)$ and non-pregnancies $(P<0.01)$ were higher than that in controls. Similar distribution of the proportions in non-pregnant cows to that of pregnant cows may have resulted because embryos kept their viability in the uterus for a period following transfer to the recipient animal. The result from Experiment 2 further indicated that the presence of embryos in the uterus during Day 7 or 8 to 14 or 15 obviously affects the dynamics of maternal platelets. Therefore, it was speculated that platelet activation in the present study may be caused by embryo-derived PAF-induced platelet aggregation $[17,18]$. A significantly transient PLT change observed in twin and single pregnant cows was unlikely to result from platelet aggregation (activation) through neither the adenosine diphosphate pathway [19] nor the cyclooxygenase-dependent pathway [20]. In the present study no effect of the number of embryos on the degree of PLT change was clearly observed. In another study mouse embryo-derived PAF activity in embryo culture medium was not altered by an increasing embryo number [21]. Adamson et al. [21] suggested that embryo-derived PAF were not strictly identical with PAF.
Other studies have shown that significant changes in absolute platelet counts could not be detected in post-fertilization between non-pregnant and pregnant inseminated heifers [9]. Also, EPAT in pregnant cows following embryo transfer could not be demonstrated. Johnson et al. [8] reported that no EPAT was observed, although significant alterations in PLT were seen, when blood was daily taken from inseminated cows. Hansel et al. [7] reported in their review that absolute PLT counts were significantly lower in pregnant than in nonpregnant heifers during Days 7 to 16 after insemination. Although the precise reason for these various results is not clear, the use of mean concentrations of absolute PLT in cattle for statistical analysis may be inappropriate because, in general, the individual difference of physiological data in conventional livestock animals must be larger than that of the inbred mice used by $\mathrm{O}^{\prime}$ Neill's original study [1].

Using relative PLT the present study could demonstrate that PLT significantly changed in association with the presence of viable embryos in the reproductive tract of cows. It was also found that the change was either a fall or a rise, depending upon cows. O'Neill et al. [16] reported that women achieving pregnancy following IVF-ET who failed to show EPAT generally displayed a significant increase in PLT. The present observation in cows was consistent with the report in humans. $\mathrm{O}^{\prime}$ Neill's group also suggested that a considerable degree of the variability in response could be related to the thrombocrit before embryo transfer. This observation appeared to be interpreted as evidence for the activation of the thrombopoietic system [16]. If an individual has a low pre-transfer thrombocrit, then a fall in PLT is observed. On the contrary, a rise in PLT is likely to occur if a cow has a high thrombocrit.

In studies with cows, including the present study, sustained alterations in PLT have not been observed, although PAF-activity has been detected in the medium in which cow embryos were cultured [22]. Johnson et al. [8] suggested that the effect of embryo-derived PAF was localized, and that the exertion of systemic effects was attenuated by the simple fact that blood volume is so great in the bovid compared to that in the mouse, even though embryo size is relatively similar. Also, the spleen of the cow may have a larger buffering effect in the dynamics of platelet activation than it 
does in the mouse. Adamson et al. [23] suggested that thrombocytopenia could be inhibited by platelet desensitization resulting from loss of platelet receptors because of their internalization following binding with subthreshold concentrations of PAF. Further study will be needed to confirm evidence of the presence of PAF in circulating blood.

Adamson et al. [23] suggested that platelet activation at the time of fertilization is important for successful implantation in mice. The present study demonstrated that establishment of pregnancy in cows, at least the presence of viable embryos in the uterus, involved maternal platelet activation. Whereas the physiological significance of platelet activation is not clear, it has been speculated that biologically active materials such as biogenic amines, histamine, serotonin, fibronectin, prostaglandins, platelet-derived growth factor and so on, known to be released upon platelet activation, may have local and systemic effects that are responsible for pregnancy establishment [1].

The present study also demonstrated that hematopoietic activation occurred in the presence of viable embryos in the uterus of cows from the results of the positive correlation between RBC and PLT in pregnant cows in Experiment 1 and the results of Experiment 2. In the human, expansion of the total red cell volume begins in the second trimester, and the greatest increase occurs in the third trimester [24]. Also, serum erythropoietin rises detectably only after the eighth week of human pregnancy [25]. Therefore, the implication of hematopoietic activation in the preimplantation stage was reported first in the present study. HCT pattern had a good correlation with RBC pattern. This means that no hemodilution associated with pregnancy occurred in the cows [26].

In conclusion, the results of the present study suggested that maternal platelet activation occurs during early pregnancy in cattle. However, platelet counts are unlikely to be a clinically useful means of early pregnancy detection in cows due to the large individual variation in the responses to this platelet activation. The implication of maternal platelet activation in the preimplantation stage of cows should be evaluated in suitability to pregnancy potential. Also, the implication of hematopoietic activation in preimplantation stage was reported first in the present study.

\section{Acknowledgments}

We greatly appreciate Dr. Chris O'Neill in Royal North Shore Hospital for the critical review, valuable discussion and encouragement on the manuscript. The authors also thank the first animal management section in NIAI for the plentiful supply and care of animals.

\section{References}

1. O'Neill C. Thrombocytopenia is an initial maternal response to fertilization in mice. J Reprod Fertil 1985; 73: 559-566.

2. $\mathbf{O}^{\prime}$ Neill C. Examination of the causes of early pregnancy-associated thrombocytopenia in mice. $J$ Reprod Fertil 1985; 73: 567-577.

3. O'Neill C. Partial characterization of the embryoderived platelet-activating factor in mice. J Reprod Fertil 1985; 75: 375-380.

4. O'Neill C, Gidley-Baird AA, Pike IL, Porter RN, Sinosich MJ, Saunders DM. Maternal blood platelet physiology and luteal-phase endocrinology as a means of monitoring pre- and postimplantation embryo viability following in vitro fertilization. $J$ in Vitro Fertil Embryo Transfer 1985; 2: 87-93.

5. Roberts TK, Adamson LM, Smart YC, Stanger JD, Murdoch RN. An evaluation of peripheral blood platelet enumeration as a monitor of fertilization and early pregnancy. Fertil Steril 1987; 47: 848-854.
6. Hearn JP, Gidley-Baird AA, Hodges JK, Summers PM, Webley GE. Embryonic signals during the peri-implantation period in primates. J Reprod Fertil Suppl 1988; 36: 49-58.

7. Hansel W, Stock A, Battista PJ. Low molecular weight lipid-soluble luteotrophic factor(s) produced by conceptuses in cows. J Reprod Fertil, Suppl 1989; 37: 11-17.

8. Johnson SK, Johnson AR, Keefer CL, Silcox RW. Blood constituents during the estrous cycle and early pregnancy in dairy cows. Theriogenology 1990; 34: 701-707.

9. Thibodeaux JK, Roussel JD, Adkinson RW, Goodeaux LL. The evaluation of pregnancy associated thrombocytopenia as an initial response to pregnancy in cattle. Theriogenology 1990; 33: 336.

10. Evans J, Gregory L, Walker S. Peripheral blood platelet counts in the management of very early pregnancy failure following gamete intrafallopian 
transfer. J In Vitro Fertiliz Embryo Transfer 1990; 7: 54-57.

11. Kuwayama M, Hamano S, Nagai T. Vitrification of bovine blastocysts obtained by in vitro culture of oocytes matured and fertilized in vitro. J Reprod Fertil 1992; 96: 187-193.

12. Kojima T, Soma T, Oguri N. The effects of simplification of donor preparation, several superovulatory treatments by FSH-P and the single insemination regimen on embryo production in Japanese beef cattle. Jpn J Zootech Sci 1989; 60: 721-727.

13. Kojima T, Soma T, Oguri N. Effect of ice nucleation by droplet of immobilized silver iodide on freezing of rabbit and bovine embryos. Theriogenology 1988; 30: 1199-1207.

14. Statistical Analysis System. SAS ${ }^{\circledR}$ User's Guide: Statistics, Version 6.03 Edition, Cary, NC: SAS Inst., Inc.; 1988.

15. Kojima T, Shimizu M, Tomizuka T. Effect of administration with low-dose FSH to recipient cows on embryonic survival after bilateral nonsurgical embryo transfer. J Reprod Dev 1995; 41: 277-286.

16. O'Neill C, Collier M, Saunders DM. Embryo-derived platelet-activating factor: its diagnostic and therapeutic future. New York Acad Sci 1988; 541: 398-406.

17. Chignard $M$, Le Couedic JP, Tence $M$, Vargaftig BB, Benveniste J. The role of platelet-activating factor in platelet aggregation. Nature 1979; 279: 799-800.

18. Liggitt HD, Leid RW, Huston L. Aggregation of bovine platelets by acetyl glyceryl ether phosphorylcholine (platelet activating factor). Vet
Immunol Immunopathology 1984; 7: 81-87.

19. Born GVR. Aggregation of blood platelets by adenosine diphosphate and its reversal. Nature 1962; 194: 927-929.

20. Vargaftig BB, Zirinis P. Platelet aggregation induced by arachidonic acid is accompanied by release of potential inflammatory mediators distinct from $\mathrm{PGE}_{2}$ and $\mathrm{PGF}_{2} \alpha$. Nature 1973; 244: 114-116.

21. Adamson LM, Podsiadly B, Smart YC, Stanger JD, Roberts TK. Studies on murine embryo-derived platelet-activating factor (EPAF). Mol Reprod Dev 1991; 30: 207-213.

22. Stock AE, Axtell R, Jones H, Jones G, Hansel W. Evaluation of human and bovine embryos by measurements of PAF in the culture medium. Theriogenology 1990; 33: 332.

23. Adamson LM, Smart YC, Stanger JD, Murdoch RN, Roberts TK. Mechanistic studies of early pregnancy associated thrombocytopenia (EPAT) in the mouse. Amer J Reprod Immunol Microbiol 1987; 13: 117-120.

24. Metcalfe J, Stock MK, Barron DH. Maternal physiology during gestation. In: Knobil E, Neill J (eds.), The Physiology of Reproduction. Raven Press Ltd, New York, 1988; 2145-2176.

25. Cotes PM, Canning CE, Lind T. Changes in serum immunoreactive erythropoietin during the menstrual cycle and normal pregnancy. Brit J Obstetr Gynaecol 1983; 90: 304-311.

26. Reynolds M. Measurement of bovine plasma and blood volume during pregnancy and lactation. Amer J Physiol 1953; 175: 118-122. 